\title{
Kind, Eltern, Stadtteil
}

\section{Das Evangelische Kinder- und Familienzentrum Goldstein in Frankfurt am Main}

\section{JEANETTE KREß}

Jeanette Kreß ist Leiterin und Koordinatorin im Evangelischen Kinderund Familienzentrum Goldstein in Frankfurt am Main. Sie ist Diplom Sozialpädagogin und hat einen Master of Arts im Management von Gesundheits- und Sozialeinrichtungen. Seit 2012 ist sie Beraterin für Early Excellence Centres.

http://diakonischeswerk-frankfurt.de

\author{
Der Early-Excellence-Ansatz verlangt eine positive \\ Grundhaltung der Fachkräfte gegenüber den Kindern \\ und Familien. Das zeigt sich unter anderem im \\ Ernstnehmen der Kompetenzen von Eltern und Kindern. \\ Wie diese Anspruch in der Praxis eingelöst werden \\ kann, zeigt die Arbeit eines stadtteilorientierten \\ Kinder- und Familienzentrums in Frankfurt am Main.
}

Kinder- und Familienzentren haben den Auftrag, niederschwellig Beratungs-, Bildungs- und Freizeitangebote für $\mathrm{Fa}$ milien anzubieten. Der Begriff »Kinderund Familienzentrum « ist nicht festgelegt, wodurch es viele Variationen mit unterschiedlichen Kooperationen gibt.

Familienzentren können zum Teil auch Einrichtungen, sowohl mit, als auch ohne Kindertageseinrichtung sein. Diese Einrichtungen bieten Kursangebote in verschiedenen Formen für $\mathrm{Fa}$ milien an. Meist liegt der Schwerpunkt der Angebote bei jungen Familien. Es können aber auch Angebote für Großeltern dabei sein. Kooperationspartner eines Kinder- und Familienzentrums sind meist Erziehungsberatungsstellen, Familienbildungsstätten, Gesundheitszentren und andere Kindertageseinrichtungen im Einzugsgebiet.

Die Stadt Frankfurt am Main hat sich zum Ziel gesetzt, in Stadtteilen mit besonderer Problemlage Kinder- und Familienzentren fachlich wie finanziell zu fördern. Voraussetzung dazu ist neben der besonderen Problemlage im Einzugsgebiet der Einrichtung, dass die Kindertageseinrichtung aus mindestens vier Gruppen besteht. Weiterhin soll die Einrichtung mit einer Familienbildungsstätte kooperieren und nach dem Early-Excellence-Ansatz arbeiten. Die Stadt Frankfurt am Main baut in der Zwischenzeit ein Qualifizierungsprogramm dazu auf; seit 2011 stellt das Sozialministerium des Landes Hessen ebenfalls Fördermittel zur Verfügung.
Im Early-Excellence-Ansatz wird nach einem spezifischen ethischen Code gearbeitet. Dieser bestimmt eine positive Grundhaltung der Fachkräfte gegenüber den Kindern, Familien sowie Mitarbeiterinnen und Mitarbeiter. Dies zeigt sich in einem offenen Zugang zu den Familien und der Einbeziehung ihrer Kompetenzen. Dazu gehört auch die Entwicklung einer gemeinsamen Sprache zwischen Fachkräften und Familien, so dass Eltern sich auf Augenhöhe angenommen fühlen und eine Vertrauensbasis aufgebaut wird.

Bedeutsam für den Early-ExcellenceAnsatz ist eine konsequente Ausrichtung auf die Wünsche und Bedürfnisse der Eltern. Damit ist gemeint, die Familien in der Umsetzung zu ermutigen, ihnen ihre Ressourcen und Stärken zu verdeutlichen und gegebenenfalls Rahmenbedingungen zur Zielerreichung zu ermöglichen. Die Entwicklung des jeweiligen Kindes wird für die Eltern dokumentiert, steht ihnen jederzeit zur Verfügung und gehört zur Grundlage der Elterngespräche (vgl. Christine Karkow, Barbara Kühnel: Das Berliner Modell. Qualitätskriterien im Early Excellence Ansatz 2008, Seite 5).

\section{Wie wir arbeiten}

Zielsetzung im Early Excellence Ansatz ist die Verbesserung der Bildungs- und Betreuungsqualität in den Einrichtungen, die Entwicklung einer ganzheitlichen Angebotsstruktur für die Familien 


\section{Was versteht man eigentlich unter dem "Early-Excellence-Ansatzu?}

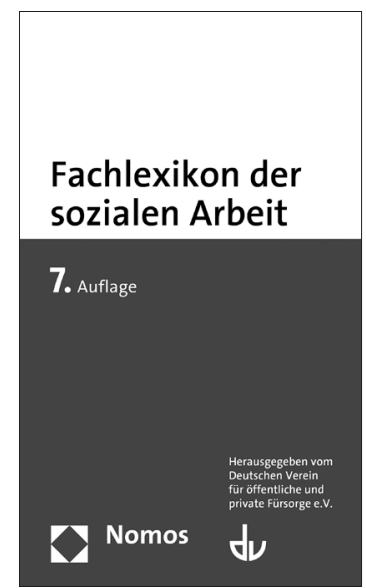

"Early Excellence " bedeutet, ressourcenorientiert alle Kinder zu fördern und Familien zu unterstützen. Leitidee der Early Excellence Centres ist die Integration der Bereiche Erziehung, Bildung und Betreu- ung, Gesundheit sowie Unterstützung und Beratung für Familien. Vor ca. 20 Jahren entstand in England die Idee, Zentren für eine kindgerechtere und effektivere Früherziehung einzurichten. 1997 rief die englische Regierung das Early-ExcellenceCentres-Programm ins Leben, dessen Ziele und Schwerpunkte in das "Sure Start Program" (2000) und das "Children Centre Program« (2004) integriert und erweitert wurden. Heute gibt es in England ca. 500 dieser frühpädagogischen Zentren. 2001 wurde in Berlin das erste deutsche Early Excellence Centre im Kinder- und Familienzentrum Schillerstraße des PestalozziFröbel-Hauses in Berlin eröffnet. Die Early Excellence Centres verstehen sich als "Gemeinschaft forschend Lernender" zu der die Kinder, ihre Familien und das pädagogische Fachpersonal gleichermaßen gehören. Im Mittelpunkt des pädagogi- schen Handelns stehen das Kind und seine Bildungsprozesse. Diese gilt es, mittels Beobachtung zu erkennen und das Kind auf hohem Niveau individuell zu fördern. Dabei stehen folgende Aspekte im Vordergrund: Wohlbefinden, Engagement und Schemata (Verhaltensmuster). Eltern, als Experten ihrer Kinder, und pädagogische Fachkräfte gehen eine neue Bildungs- und Erziehungspartnerschaft ein, in der die Eltern gleichberechtigt in die Diskussion um Schlüsselkonzepte der kindlichen Entwicklung einbezogen werden und bei der Förderung ihrer Kinder mitarbeiten.

Maria-Theresia Münch

Quelle: Deutscher Verein für öffentliche und private Fürsorge e. V. (Hg.): Fachlexikon der sozialen Arbeit. 7. Auflage. Nomos Verlagsgesellschaft, Baden-Baden 2011. 1.139 Seiten. 44,- Euro. ISBN 978-3-8329-5153-5. im Stadtteil und die Einbeziehung der Eltern in die Bildungsprozesse ihrer Kinder. Die Umsetzung dieser Grundhaltung und deren Ziele ist eine Aufgabe im Evangelischen Kinder- und Familienzentrum Goldstein.

Das Evangelische Kinder- und Familienzentrum Goldstein besteht seit November 2009 und entwickelte sich aus einer Evangelischen Spiel- und Lernstube. Die Spiel- und Lernstube bestand fast drei Jahrzehnte und betreute in einer Fünf-Zimmer-Wohnung 15 Kindergarten- und 12 Hortkinder. Die Mitarbeiterinnen arbeiteten schon damals eng mit den Eltern und dem Stadtteil zusammen.

Auf diese Arbeit konnte das Evangelische Kinder- und Familienzentrum Goldstein aufbauen, das mit seiner Kinderbetreuung in einen Neubau einzog. Die Evangelische Familienbildung begann mit ihrer Aufgabe im Sommer 2010. Ab diesem Zeitpunkt starteten die ersten Angebote. Im Mai 2011 zog die Familienbildung in die ehemaligen Räume der Spiel- und Lernstube ein. Seit diesem Zeitpunkt können nun Beratungs-, Bildungs- und Freizeitangebote weiter ausgebaut werden.

Beide Räumlichkeiten liegen sehr nahe beieinander. In der Einrichtung betreuen wir in jeweils zwei Krabbelstuben- und Kindergartengruppen insgesamt 64 Kinder. Die Goldsteiner Bevölkerungsstruktur mit einem hohen Anteil an Familien mit Migrationshintergrund spiegelt sich in der Belegung der Einrichtung wider.
Zunächst ging es um den inhaltlichen Aufbau der Kindertageseinrichtung sowie um eine Zusammenarbeit mit der Evangelischen Familienbildung. Schwerpunkt lag zu Beginn im Zusammenwachsen zu einer Einrichtung, Klärung der Aufgabenaufteilung und der gemeinsamen Entwicklung der Angebote. Eine besondere Aufgabe bestand im Aufbau der Kommunikationswege zwischen beiden Arbeitsbereichen, die Einbeziehung der Erzieherinnen und Erzieher in diesen Prozess und die Öffentlichkeitsarbeit.

Goldstein ist kein eigener Stadtteil von Frankfurt am Main, sondern gehört zum Stadtteil Schwanheim. In den 1970erJahren entstand die Hochhaussiedung »Im Heisenrath «. Goldstein besteht aus Siedlungs- und individuellen Einfamilienhäusern sowie aus Mehrfamilien- und Hochhäusern mit Sozialwohnungen. Die Infrastruktur ist nur schwach entwickelt. Es gibt kaum Einkaufsmöglichkeiten und die Anbindung durch den öffentlichen Nahverkehr ist schlecht.

In Goldstein leben viele Familien mit Migrationshintergrund. Ebenfalls erhalten hier viele Familien existenzsichernde Sozialleistungen. In Goldstein gibt es fünf Kindertageseinrichtungen, ein Kinder- und ein Jugendhaus, eine Grundund eine Gesamtschule, verschiedene Beratungsstellen mit unterschiedlichen Schwerpunkten sowie mehrere Vereine. Für die Belange der Familien setzen sich verschiedene Arbeitsgruppen mit jeweils eigenen Schwerpunkten ein. Dies können die Anliegen der Kinder und der Familien sein, präventive Arbeit, Übergänge gestalten und fachliche Angebote. Ein hohes Engagement geht besonders von einigen Goldsteiner Bürgerinnen und Bürger aus, die auch in sozialen Einrichtungen im Stadtteil arbeiten.

Eine weitere Herausforderung war die Kontaktaufnahme zum Stadtteil. Konkret bedeutete dies, die Teilnahme an verschiedenen Arbeitsgruppen, Kontaktaufnahme zu den sozialen Einrichtungen, Erhalt eines Überblickes über die Angebote und aktive Mitarbeit im Stadtteil. Parallel dazu gingen wir gemeinsam in die Einrichtungen und thematisierten den Bedarf in Goldstein und die eigenen Möglichkeiten der Umsetzung. Es ging hierbei auch um den Aufbau von Kooperationen und um eine Abgrenzung der Angebote, zur Vermeidung einer Konkurrenz.

Zu einer gelungenen Kooperation gehört zunächst eine wertschätzende Haltung zum Kooperationspartner. Dies gilt besonders für die bis jetzt geleistete Arbeit im Stadtteil und den einzelnen Kooperationspartnern. Weitere Hilfestellung ist ein regelmäßiger Austausch, gegenseitige Hilfe, Konkurrenz in den Angeboten vermeiden indem klare Absprachen stattfinden und Rahmen zu den einzelnen Angeboten abzustecken. Die positive Zusammenarbeit unter den einzelnen Einrichtungen und in den Arbeitsgruppen zeigt sich in kurzen Informationswegen, gegenseitige Hilfe, gemein- 
same Planung und Durchführung von Angeboten für den Stadtteil oder auch mal eine Hospitation in einer anderen Einrichtung. Bezüglich der Wünsche und Bedürfnisse der Eltern sprachen wir diese persönlich an. Daraus entwickelten sich die ersten Angebote im Kinder- und Familienzentrum Goldstein.

Mit der Zeit vergrößerten sich für das Kinder- und Familienzentrum Goldstein der Sozialraum und die Kontakte. Neben den vom Frankfurter Stadtschulamt organisierten Treffen der Kinder- und Familienzentren wurden wir Gründungsmitglied im Bündnis West des Bündnisses für Familien in Frankfurt am Main. Ebenfalls nehmen wir an den Netzwerktreffen Hessischer Kinder- und Familienzentren teil.

Im Kinder- und Familienzentrum Goldstein wird schon bei der Anmeldung zu einem Krabbelstuben- oder Kindergartenplatz großen Wert auf eine ausführliche Beratung und eine erste Hilfestellung bei einer Platzsuche gelegt. Wie sieht die aktuelle Situation im Stadtteil aus? Wo finde ich weitere Adressen? Wo finde ich die Liste für freigemeldete Plätze? Fragen zum Konzept, die Vorstellung der Angebote des Zentrums und die Beantwortung von individuellen Fragen sind Inhalte des Anmeldegesprächs. Zu diesem Zeitpunkt erhalten wir schon den ersten Eindruck über die Lebenssituation der Familien.

Ein weiterer guter Einstieg in die Zusammenarbeit mit den Eltern bietet unser wöchentlich stattfindendes Café. Hier kann niederschwellig über Alltagssituationen der Familie gesprochen und beraten werden. Wichtig ist im Alltag der Einrichtung ein offenes Ohr für die Familien zu haben. Im Dialog zwischen Fachkräften und Eltern erkennen wir die Problematik und die Wünsche nach Hilfestellung. Daraus können sich dann Angebote entwickeln. Das Café, wie auch Bildungs- und Beratungsangebote, gekoppelt mit dem Angebot einer Kinderbetreuung, werden gerne von den Fami- bogens gehörte eine Beurteilung der aktuellen Angebote der Einrichtung, eine Abfrage nach weiteren Beratungs-, Bildungs- und Serviceleistungen. Von unserer Seite wurden Vorschläge eingebracht. Diese Möglichkeit bestand natürlich auch für die Eltern. Ebenfalls beinhaltete der Fragebogen die Frage, welche Stärken die Eltern anderen

\section{"Gefragt sind kostengünstige und niedrigschwellige Angebote»}

lien angenommen. Die Kinderbetreuung übernehmen Mütter aus dem Stadtteil in ihrer Funktion als Ehrenamtliche.

Gerne angenommen werden niederschwellige Angebote, die kostenlos oder kostengünstig sind. Hierzu zählen das Café, gemeinsames Kochen oder ElternKind-Angebote wie gemeinsames Basteln. Eine weitere Nachfrage besteht nach Sprachangeboten bis hin zur Alphabetisierung. Bewegungsangebote und die Sozialberatung sind ebenfalls gefragt.

\section{Wünsche erfragen, Prioritäten festlegen, Angebote kontinuierlich verbessern}

Zur Umsetzung des Early-ExcellenceAnsatzes gehört, Wünsche und Bedarfe der Eltern zu ermitteln und die Einbeziehung der Eltern in die Umsetzung dieser Angebote. So fand im Herbst 2012 die erste Elternbefragung im Kinder- und Familienzentrum Goldstein statt. Zum Inhalt des Elternfrage-
Familien gegenüber zur Verfügung stellen möchten. Auch interessierte uns die Frage, wie die Eltern die Zusammenarbeit mit uns beurteilen, wie weit sie in die Arbeit mit einbezogen sind und wie ihre Einschätzung dazu ist, dass sie ermutigt wurden, ihre Wünsche, Ideen und Kritik zu äußern. Wir hatten einen guten Rücklauf und erhielten positive Rückmeldungen.

Bei der Auswertung der Befragung ergab sich eine Prioritätenliste, an deren Umsetzung wir nun arbeiten. Eine hohe Nachfrage der Eltern bestand zu den Themen Erziehung, Ernährung, Zahnpflege, Elternstammtisch, Ausflüge mit Eltern und Kindern, Flohmarkt, Bauund Abenteueraktionen für Väter mit Kindern, Rückengymnastik für Frauen wie auch der Wunsch nach einem Alphabetisierungskurs. Um das Angebote zur Rückmeldung zu unserer Arbeit zu erweitern, führten wir neben den schon bestehenden persönlichen Gesprächen, den regelmäßigen Angeboten beim El-

\section{Arbeitshilfe: Die Kindertagesbetreuung in der wirtschaftlichen Jugendhilfe}

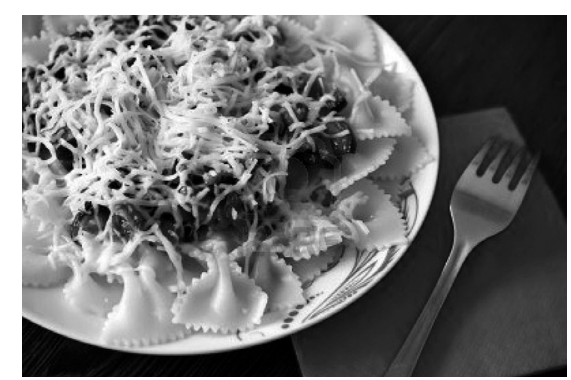

"Wer trägt die Kosten für ein warmes Mittagessen?« ist eine der vielen Fragen, die in der Praxis gestellt werden. Die Antworten richten sich oft nach Empfehlungen oder Richtlinien oder Dienstanwei- sungen, werden aber nicht systematisch aus dem Gesetz abgeleitet. Systematisch muss unterschieden werden zwischen der Förderungsleistung nach den $\S \S 22$ bis 24 SGB VIII, insbesondere ihrem Umfang und ihrem Verbindlichkeitsgrad; dem Kostenbeitrag, der für diese Leistung erhoben werden kann ( $\$ 90$ Abs. 1 SGB VIII) und der Übernahme dieses Kostenbeitrags ( $\$ 90$ Abs. 3 SGB VIII). Die Übernahme des Kostenbeitrags wiederum hängt von Fragen der Einkommensberechnung nach dem SGB XII ab. Erhalten die Eltern Leistungen auch nach anderen Sozialgesetzen, ist fraglich, ob diese Leistungen als Einkommen einzusetzen sind oder ob solche Leistungen vorrangig vor der Jugendhilfe zu erbringen sind ( $\$ 10$ SGB VIII). Als Folge ergibt sich dann die Kostenerstattung nach $\S 104$ SGB $X$. Das Zusammenspiel der Regelungen in den verschiedenen Sozialgesetzbüchern macht der Praxis erhebliche Schwierigkeiten. Oftmals werden auch die Änderungen des SGB VIII durch TAG (2004) und KiföG (2008) nicht berücksichtigt.

Quelle: Peter-Christian Kunkel: Kindertagesbetreuung. Diskussionspapiere Nr. 2012-07 der Hochschule für öffentliche Verwaltung. www.hs-kehl.de/DE/Hochschule/Forschung/Forschungsergebnisse/ Forschungsarbeiten/2012-07.pdf 
ternabend zur Einbringung eigene Anliegen, noch den Elternbriefkasten für Rückmeldungen ein.

Trotz der Befragung nach Wünschen und Interessen kommt es immer wieder vor, dass Angebote nicht angenommen oder die Teilnahme gering ist. Wir versuchen dann Ursachen zu erforschen und falls möglich, die Rahmenbedingungen zu verändern. Wichtig dabei ist sowohl einen Vertrauensaufbau zur leitenden Person als auch mit den Eltern den zeitliche Rahmen der jeweiligen Angebote abzustimmen. Es ist not- wendig, immer wieder zu reflektieren, sich Rückmeldung geben zu lassen und sich durch ein Scheitern von einzelnen Angeboten nicht entmutigen zu lassen. Hilfreich dabei ist der Austausch mit anderen Kinder-und Familienzentren.

Ein weiterer Schwerpunkt nach dem Early-Excellence-Ansatz ist die Beobachtung und Dokumentation der Entwicklung der Kinder. So sind wir derzeit dabei, in der Kindertagesstätte Portfolios einzuführen. Wir legen unseren Schwerpunkt dabei auf Lerngeschichten und Beobachtungen der Kin- der. Die Eltern werden beteiligt, indem sie Familienbilder mitbringen oder die Stärken ihrer Kinder im Portfolio dokumentieren. Die Portfoliomappe ist Grundlage der Entwicklungsgespräche.

Unsere Ziele für die nächste Zeit sind die weitere Umsetzung des EarlyExcellence-Ansatzes im Alltag mit den Kindern, Eltern und Mitarbeitenden, die Verankerung von Portfolios mit den Lerngeschichten und der Ausbau der Angebote unter Einbeziehung von Ehrenamtlichen.

\section{Altern in Europa}

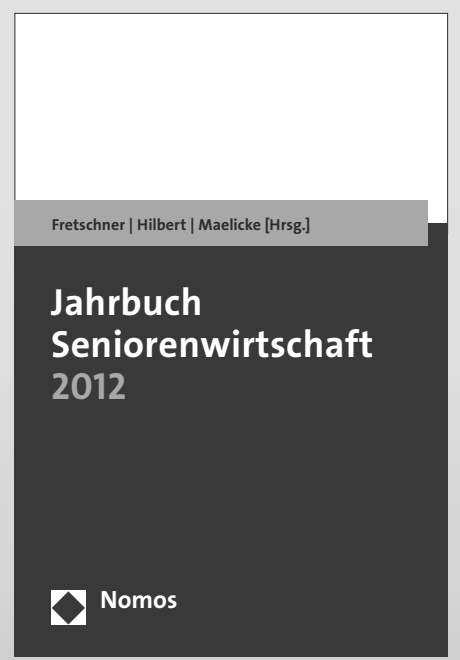

Die Europäische Union hat das Jahr 2012 zum Europäischen Jahr für aktives Altern und die Solidarität zwischen den Generationen erklärt. Damit wird einerseits der demographischen Entwicklung in Europa Rechnung getragen und anerkannt, dass die Zukunft Europas nur durch die aktive Gestaltung des demographischen Wandels gewährleistet werden kann. Auf der anderen Seite wird durch die besondere Betonung der

\author{
Jahrbuch \\ Seniorenwirtschaft 2012 \\ Herausgegeben von \\ Prof. Dr. Rainer Fretschner, \\ PD Dr. Josef Hilbert und \\ Prof. Dr. Bernd Maelicke \\ 2012, 122 S., brosch., 29,-€ \\ ISBN 978-3-8487-0037-8
}

Solidarität zwischen den Generationen auf die Notwendigkeit einer gerechten Lastenverteilung zwischen den Generationen aufmerksam gemacht.

Das Jahrbuch ist konzipiert als Kompendium zu aktuellen Entwicklungen und Fragestellungen, mit denen sich die Akteure aus der Seniorenwirtschaft auseinandersetzen müssen und die Impulse für die Weiterentwicklung geben können. 